Poster 22

The Effects of Underwater Treadmill Therapy on the Rehabilitation of a Polytrauma Patient: A Case Report

Anthony E. Urbisci, MD (James A. Haley Veterans' Hospital, Tampa, FL, United States), Adam H. Rawji, BS, Aaron J. Martin, DO, Barbara Darkangelo, DPT, Cory Keirn, DPT

Disclosures: Anthony Urbisci: I Have No Relevant Financial Relationships To Disclose

Case/Program Description: The patient is a 30 -year-old man status post motor vehicle accident who was ejected from his vehicle. He sustained multiple fractures of his extremities and spine which required multiple surgical corrections. Once medically stable, the patient's therapy regimen started with 2 months of land based physical therapy before underwater treadmill therapy (UTT) was incorporated. Based on prior studies of UTT, the following functional outcome measures were recorded throughout the rehabilitation process: heart rate, blood pressure, pain scores, range of motion, manual muscle testing, and two-minute walk tests. To account for the concurrent land based therapy, the rate of improvement of each outcome measure was noted before and after the initiation of underwater treadmill therapy. Setting: Polytrauma inpatient rehabilitation unit at a veterans' hospital.

Results: Based on the data collected, the patient showed an appreciable change in pain scores after initiation of UTT. His left ankle dorsiflexion also improved significantly after initiation of UTT, improving from $0^{\circ}$ to $15^{\circ}$. The rate of improvement of manual muscle strength, range of motion of other joints, and two-minute walk tests did not change once UTT was incorporated. His heart rate and blood pressure remained unchanged during the admission. Subjectively, the patient and his therapists noted improvement in gait and improved exercise tolerance with subsequent UTT sessions. Significant reduction in left knee, left hip, and back pain was also reported.

Discussion: UTT is not a very well studied area of rehabilitation and may be a useful adjunct to traditional land based therapy to assist with reduction of pain and improvement of function.

Conclusions: UTT may be a useful form of therapy to assist with the rehabilitation of polytrauma patients.

Level of Evidence: Level V

\section{Poster 23}

An Unusual Cause of Leg Pain in a Stroke Patient: A Case Report

James G. Liadis, MD (Washington Hospital Center/Georgetown University, Washington, DC, United States)

Disclosures: James Liadis: I Have No Relevant Financial Relationships To Disclose

Case/Program Description: A 49-year-old man with a history of HTN and IDDM with resultant ESRD, peripheral neuropathy and a 2-year history of lacunar infarct with residual right hemiparesis who had recently undergone a renal and pancreatic transplantation presents to the emergency department with a 2-week history of right lower extremity pain and swelling. The patient had noted no history of trauma with insidious calf erythema and pain. He noted significant warmth and severe pain which worsened with any movement of the leg.

Setting: Academic Teaching Hospital.

Results: Due to the patient's hemiparesis, initial concern was for DVT which was ruled out with Doppler ultrasound. MRI of the lower extremity showed non-specific edema within and overlying an area of the gastrocnemius, concerning for myositis or another infectious process especially with the patient's immunosuppressive regimen. ID was consulted and several antibiotics were administered without symptomatic improvement. Due to the exquisite tenderness with range of motion of the gastroc-soleus muscle complex, compartment pressure testing was done and ruled out compartment syndrome. Repeat MRI again revealed feathery edema within and superficial to the gastrocnemius. The decision was made to perform a biopsy. Skin biopsy showed non-specific chronic stasis changes while muscle biopsy revealed muscle necrosis in the setting of small vessel ischemia consistent with a diagnosis of diabetic muscle infarct.

Discussion: Diabetic muscle infarct is a rare complication of poorly controlled diabetes which occurs in patients with long standing diabetes, usually type I. It typically presents as swelling and tenderness of the involved muscles with the thigh and calf being the most frequently affected regions. There are case reports similar to this case in which diabetic muscle infarct can occur even after pancreatic transplant as a result of the significant microvascular damage already present.

Conclusions: Diabetic muscle infarct is an important diagnostic consideration in the appropriate patient after ruling out other more common causes of leg pain and swelling.

Level of Evidence: Level $\mathrm{V}$

\section{Poster 24}

The Role of the Spinal Cord Injury Care Provider in Safeguarding Patient's Best Interests and Values: A Case Report and Discussion in Palliative Wound Care

Diya Goorah, MD (SUNY Upstate Medical University/Syracuse, Syracuse, New York, United States), Allan M. Landes, PhD, Gizelda T. Casella, MD, PhD, Sarah Laue, RN, CWOCN, Dorin E. Laufer, LMSW, CASAC, Stephen Lebduska, MD

Disclosures: Diya Goorah: I Have No Relevant Financial Relationships To Disclose

Case/Program Description: We present a process to resolve a bioethics dilemma: spinal cord injury $(\mathrm{SCl})$ patient with healable wound where Health Care Proxy (HCP) is declining care. Elderly patient with paraplegia, stage 3 sacral wound, fecal incontinence. Patient agreed to colostomy then refused after input from family member $(X)$ who had financial conflict of interest (COI). The patient was discharged to a nursing facility where HCP was changed to $X$. Patient was readmitted one month later, with worsening wounds, dehydration, urinary infection and weight loss. Team again recommended colostomy and feeding tube. The patient lacked capacity and decisions were deferred to $X$, who refused all surgical procedures, raising concerns about $\mathrm{COI}$ and adequacy of the surrogate. We describe the process for resolving this conflict.

Setting: Inpatient.

Results: The treatment team formed a multidisciplinary group of physicians, psychologist (bioethics committee member), wound care nurse, and social worker. SCI Team held family meeting with wife, former HCP, and X. Providers' use of photographs was effective in helping family to accept the need for colostomy and feeding tube. The family demonstrated understanding but remained adamant that patient's wishes were to refuse surgical procedures. Based upon the additional information, the team was convinced that this decision was based on the patient's values.

Discussion: Providers need to validate the suitability of a HCP, especially when a $\mathrm{CO}$ is identified or essential care is refused, and before establishing a palliative wound care program. We recommend the following process: 1- Use of multidisciplinary team; 2- Multiple contacts with family to establish rapport and understanding of patient's wishes; 3- Gathering data on patient's past healthcare statements; 4Family meeting to process concerns and facilitate decision making. Conclusions: A multidisciplinary team will help and reduce the sense of personal responsibility that providers feel when asked to withhold essential medical care.

Level of Evidence: Level $\mathrm{V}$

\section{Poster 25}

Predicting Return to Medical Service Among

Dysvascular Lower Extremity Amputees on an Acute Inpatient Rehabilitation Unit

Raymond Cheng, MD (University of Michigan, Ann Arbor, Michigan, United States) 
Disclosures: Raymond Cheng: I Have No Relevant Financial Relationships To Disclose

Objective: To test the hypothesis that factors indicative of chronic disease, infection risk, or poor wound healing, present at the time of admission, will be associated with 1) a higher incidence of acute transfers to inpatient medical and surgical services and 2) fewer gains in function during a course of acute inpatient rehabilitation.

Design: Cross-sectional retrospective study.

Setting: Acute inpatient rehabilitation service at a large university hospital.

Participants: 71 dysvascular amputee patients over the age of 18, admitted to the acute inpatient rehabilitation unit at the University of Michigan from 1/2011 to 4/2015.

Interventions: Not applicable.

Main Outcome Measures: Occurrence of transfer to acute service and Functional Independence Measure (FIM) total score.

Results: Logistic regression analysis and general linear regression analysis were used to test a predictive model of age, creatinine, hemoglobin, white blood cell (WBC) count, hemodialysis use, wound vac use, IV antibiotic use, and presence of a prior amputation at the time of hospital admission. No predictors were significantly associated with study outcomes.

Conclusions: These comorbidities were not significantly associated with the rate of transfer back to acute service or functional gains during inpatient rehabilitation. Future analysis should factor in the presence of diabetes and determine if a combination of these comorbidities contributes to medical complications and functional gains.

Level of Evidence: Level II

\section{Poster 26}

A Randomized Trial of Cardiac Rehabilitation for Adolescents and Adults with Congenital Heart Disease

Ana Ubeda Tikkanen, MD, PhD (Spaulding Rehabilitation Hospital, Boston, MA, United States), Jonathan Rhodes, MD, Micheal Landzberg, Ami Bhatt, MD, MPH, David M. Systrom, MD, Aaron Waxman, MD, Lilamarie Moko, BA, Robin Bradley, BS, Scott Crouter, PhD, Alexander Opotowsky, MD, MPH

Disclosures: Ana Ubeda Tikkanen: I Have No Relevant Financial Relationships To Disclose

Objective: To describe the effects of Cardiac rehabilitation (CR) programs on exercise capacity and quality of life in adolescents and adult with Congenital Heart Disease (CHD).

Design: We performed a prospective, randomized, controlled trial (NCT01822769).

Setting: Tertiary care hospitals.

Participants: Patients with moderately or severely complex $\mathrm{CHD} \geq 15$ year-old, with resting $\mathrm{O}_{2}$ saturation $\geq 91 \%$ and peak $\mathrm{O}_{2}$ consumption $\left(\mathrm{VO}_{2} \mathrm{pk}\right) \leq 80 \%$ predicted were randomized to cardiac rehabilitation or standard of care (SOC).

Interventions: 12 week, twice a week, standardized clinical CR program including exercise training and education.

Main Outcome Measures: We assessed exercise capacity, inert gas rebreathing cardiac output, quality of life, self reported health status, and other variables at baseline and after 3 months.

Results: We enrolled 28 subjects (mean age $41.1 \pm 12.1$ y, $50 \%$ male), with 13 randomized to CR and 15 to SOC. BMl averaged $28.0 \pm 3.8 \mathrm{~kg} / \mathrm{m}^{2}$ with $\mathrm{VO}_{2} \mathrm{pk}=16.8 \pm 3.8 \mathrm{ml} / \mathrm{kg} / \mathrm{min}$, peak work rate $=95 \pm 28 \mathrm{~W}$ and median Minnesota Living with Heart Failure Questionnaire (MLHFQ) score $=27$ [IQR 11-44]. CR subjects were older $(48 \pm 9 \vee 36 \pm 12$ y, $P=.01)$ but there were no significant between group differences in sex, height, weight, $\mathrm{VO}_{2}$ pk, peak work rate, cardiac output, MLHFQ score or other variables. There were no adverse events related to $C R$. Those assigned to CR lost weight over the intervention relative to SOC $(-2.3 \mathrm{~kg}, P<.03) . \mathrm{VO}_{2} \mathrm{pk}$ increased in the CR group compared with SOC $(+2.2 \mathrm{~mL} / \mathrm{kg} / \mathrm{min}, 95 \% \mathrm{Cl}$ 0.7-3.7, $P=.002$, age adjusted $+2.7 \mathrm{~mL} / \mathrm{kg} / \mathrm{min}, P=.004$; absolute increase $119 \mathrm{~mL} / \mathrm{min}, P=.01)$; there was also a nonsignificant improvement in work rate $(+8.1 \mathrm{~W}, P=.13)$ and MLHFQ score $(-6.5, P=.13)$. Among $\mathrm{n}=25$ with baseline $\mathrm{MLHFQ}>5$, there was a clinically important $>5$ point decrease in $72.7 \%$ of CR subjects compared with $28.6 \%$ of SOC subjects, $P=.047)$. Patients in the CR group also had greater improvement in a visual analogue scale self-assessment of overall health $(P<.04)$

Conclusions: $\mathrm{CR}$ is safe in adults with $\mathrm{CHD}$ and is associated with improvement in aerobic capacity and self-reported health status compared with SOC. Further studies should evaluate the longer term effects of CR in adults with CHD.

Level of Evidence: Level I

Poster 27

Acupuncture Treatment for Levator Ani Syndrome: A Case Report

Hamad T. Saleemi, DO (Stony Brook University Hospital, Port Jefferson, NY, United States), Henna Farooque, DO, Sunia Choudhury, Jun Zhang, MD

Disclosures: Hamad Saleemi: I Have No Relevant Financial Relationships To Disclose

Case/Program Description: Levator ani syndrome is a painful condition due to spasm of the levator ani muscle. The etiology is unknown and diagnosis is by exclusion of other more serious conditions such as diverticulitis, bleeding, abscess, or cancer. Treatment typically consists of muscle relaxants, warm baths, therapeutic ultrasound and in extreme cases, botox injections. Acupuncture treatment has not been studied in patients with levator ani syndrome. In our case, a 64-year-old male presented after left intraparenchymal hemorrhage status post apollo procedure and then transferred to inpatient rehabilitation. During patient's stay, he began to complain of "deep rectal pain" that was unrelieved by hemorrhoidal suppository and prevented him from participating in rehabilitation sessions. A complete work up including, CAT scan, sigmoidoscopy, and MRI were completed but without any significant findings. Patient was diagnosed with levator ani syndrome. He was started on valium suppositories which did not relieve his pain. A strict bowel regimen somewhat alleviated his symptoms. Patient reported pain that was an average of $7 / 10$ in severity and on some days it was severe enough to prevent him from participating in therapy. Patient was treated with acupuncture: Du 21, HN 3, RN16, RN 12, RN10, RN7, Bilateral LI4, LI11, SP6, ST $36 \times 60$ minutes for a total of 3 treatment sessions.

Setting: Community hospital inpatient rehabilitation unit.

Results: After his first session of acupuncture, the patient reported immense relief. He had two more acupuncture sessions thereafter. His pain rating was down to a $2 / 10$. His pain was relieved and he was able to continue acute rehabilitation.

Discussion: Although levator ani syndrome is a benign condition, the pain and discomfort it causes can become unbearable. In our case, it affected the patient's ability to participate in rehabilitation. Medication management along with botox injections have been the mainstay of treatment but with unreliable effect.

Conclusions: This case showed that acupuncture treatment successfully relieved symptoms of levator ani syndrome. However, additional research with better design and sample size is needed to identify the best treatment options for levator ani syndrome.

Level of Evidence: Level $\mathrm{V}$

\section{Poster 28}

Steroid-Induced Myopathy from Endogenous Hypercortisolism Secondary to Adrenocortical Carcinoma: A Case Report

Rajiv D. Reddy, MD (Rehabilitation Institute of Chicago/Northwestern University, Chicago, IL, United States), Gayle R. Spill, MD

Disclosures: Rajiv Reddy: I Have No Relevant Financial Relationships To Disclose

Case/Program Description: A 56-year-old female presented to the emergency department with tremulousness and muscle twitching. She had a history of newly diagnosed diabetes mellitus (DM), rapid weight 